\title{
A Robust 3D Scanning Technique for SEM
}

\author{
Grigore Moldovan ${ }^{1}$
}

1. point electronic GmbH, Erich-Neuß-Weg 15, D-06120 Halle, Germany

3D scanning is a popular technique in light-based instruments, driven primarily by the need to model and analyze shapes in 3D, however electron beam-based instruments have not yet been able to claim a significant role in this new advance. This work proposes and demonstrates a robust SEM technique that uses the same principles, but covers the micron range that is beyond the limit of optical 3D scanners.

There is a long tradition of 3D reconstruction algorithms in SEM, such as photogrammetry [1-2] and stereo-photogrammetry [3], however the approach has not found wide adoption because of limits in the techniques of the time. Instead, $2 \frac{1}{2} \mathrm{D}$ topographic reconstruction has taken focus and it is now a common SEM technique [4], however full 3D information is still needed. The related 3D technique in FIB-SEM can provide a 3D model of outer surface, but it requires an ion-beam and it is prohibitively complex and inefficient when only external shape is required. Finally, 3D TEM techniques cannot be applied to micron-sized objects because of absorption limits.

Firstly, BackScattered Electron (BSE) signal is proposed here as a standard input for 3D scanning, as the data is free of shadowing artefacts and more resilient to charging as compared with Secondary Electron (SE) signal. Secondly, a nanotip probe is proposed as the support to the micron-sized objects to be scanned, as this provides a wider range of angles for scanning and improves the reliability of reconstruction. Thirdly, simultaneous acquisition of further signals is proposed, such that additional texture(s) may be applied on the 3D model.

Portland cement powder is used here for demonstration, as it has particles of complex 3D shapes. This was loaded on a Tungsten nanotip, which was placed vertically on the stage of an ZEISS DSM982 FEG SEM upgraded with point electronics $\mathrm{GmbH}$ electronics. The stage was tilted so that the e-beam has a $60^{\circ}$ view to the nanoprobe, and the nanoprobe was placed in the middle of the SEM stage, such that $360^{\circ}$ rotation is practical. The motorized stage provided automated click-and-move repositioning.

A quadrant Si backscatter electron detector as used to acquire BSE images, where shadowing artefacts were removed by ensuring that all quadrants have the same response. Relatively low-resolution images of only 1,024x1,024 pixels were used for scanning. BSE, SE and in-lens SE signals were recorded simultaneously. The sample was rotated with $15^{\circ}$ steps, with repositioning and refocusing at each step acquisitions settings were not changed. All operations were done using the BSE signal, as this provided the highest signal-to-noise ratio and highest compositional and topographic contrast.

The BSE data is relatively free of shadowing and charging artefacts as the BSE detector is insensitive to electrons of energy less than $\sim 2 \mathrm{keV}$. The SE data show very significant shadowing and charging, as the particles are not coated and the SE detector collects very efficiently the low energy electrons affected by the charge build up. The in-lens SE data is noisy for these acquisition settings, and again shows significant edge effects and charging. A note is made that charging may be prevented be applying a conductive coating to the sample, however this is undesirable as it introduces coating artefacts. Alternatively, an Environmental SEM (ESEM) may be used to prevent charging of uncoated specimens. 
The 3D scan data was loaded into Agisoft PhotoScan photogrammetric software, and it was reconstructed following the automated workflow of aligning the photos, building dense cloud points, mesh and texture. 3D reconstruction was fully automated, except for camera calibration which required input of scale. BSE data presented no difficulties in registration and reconstruction, however charging artefacts in SE and in-lens SE data do not allow for a successful reconstruction (not shown here).

The reconstruction constructed a very dense point cloud, and therefore a very detailed mesh surface. Resolution in reconstructed model was found to be just under that of the original data (analysis not shown here). SE or in-lens SE texture may be built over the reconstructed BSE model (not shown here), as well as any other signal recorded simultaneously, such as EDS. 3D model and UV texture data may be exported for shape analysis, however this is beyond the purpose of this publication.

This work lays out the simplest and most robust approach to 3D scanning in SEM to date.

\section{References:}

[1] A Boyde and H F Ross, The Photogrammetric Record 8 (1975), p. 408

[2] L C Gontard et al., Ultramicroscopy 169 (2016), p. 80

[3] A Boyde, Journal of Microscopy 98 (1973), p. 452.

[4] M Hemmleb et al., European Microscopy Congress (2016), p. 489.
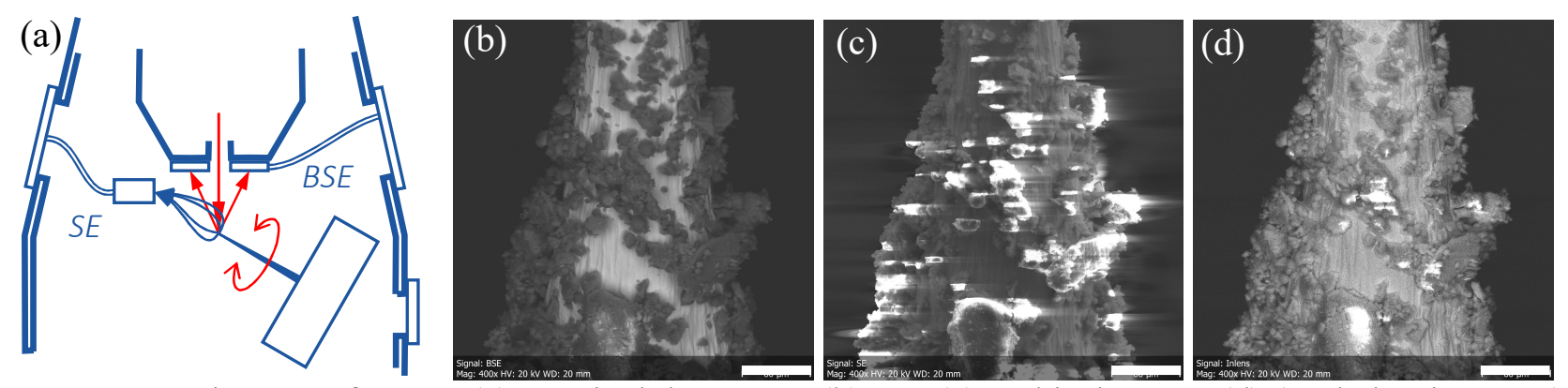

Figure 1. Diagram of set-up (a). Typical data: BSE (b), SE (c) and in-lens SE (d) (scale bar is $60 \mu \mathrm{m})$.

(a)

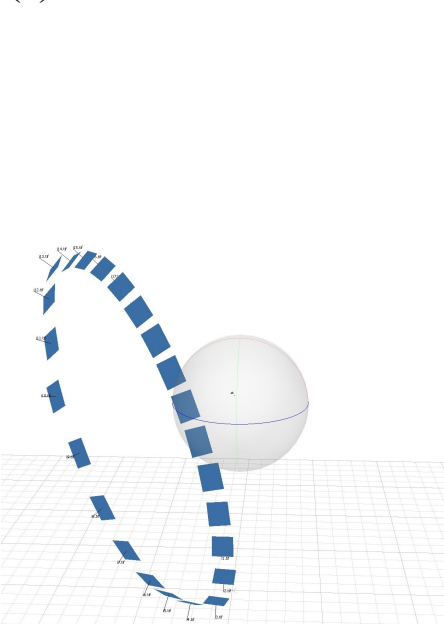

(b)

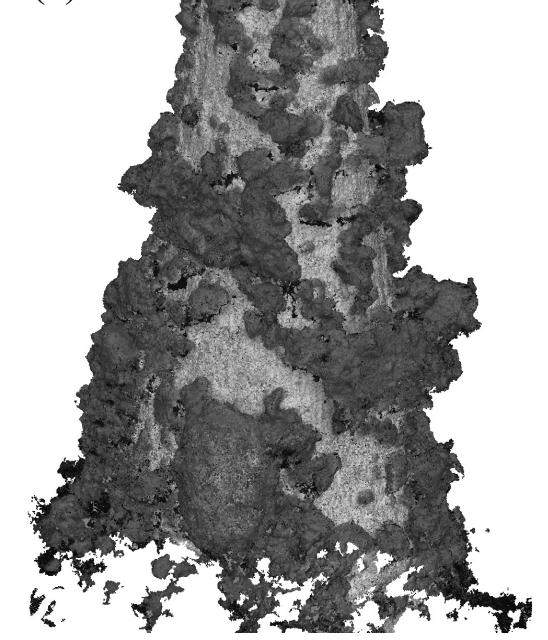

(c)

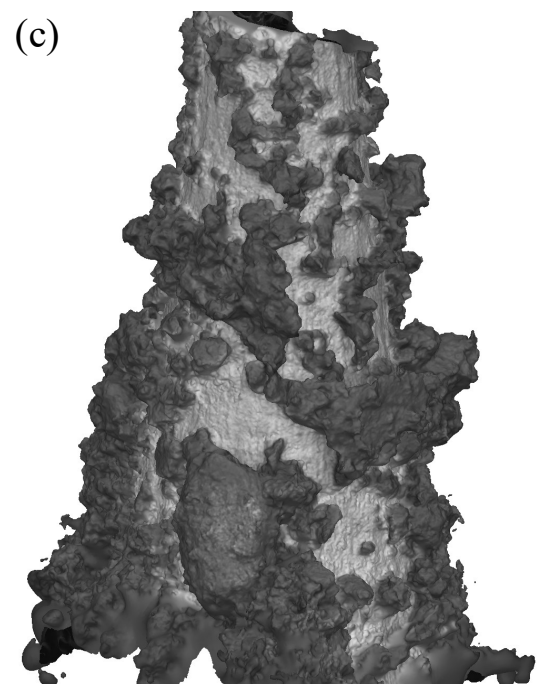

Figure 2. Automated camera positions (a), and reconstructed point cloud (b) and mesh (c). 